LPHEA /00-06

UFR-HEP 00/24

November 2000

\title{
On the Dilaton and the Axion Potentials
}

\author{
M. Chabab $\stackrel{1.2 *}{,}$ N. El Biaze ${ }^{1}$, R. Markazi ${ }^{1}$ and E.H. Saidi ${ }^{1}$ \\ ${ }^{1}$ Lab/UFR High Energy Physics, Faculty of Science, P. O. Box 1014 , Rabat, Morocco. \\ 2 LPHEA, Physics Department, Faculty of Science-Semlalia, P. O. Box 2390, Marrakesh, Morocco.
}

October 25, 2018

\begin{abstract}
We extend the Vecchia-Veneziano-Witten (VVW) model of QCD in the chiral limit and for large colour number $N_{c}$, by introducing an effective dilaton-gluon coupling from which we derive both the axion and dilaton potentials. Furthermore, using a string inspired model, we determine a new interquark potential as a perturbative series in terms of the interquark distance $r$. Our potential goes beyond Dick one obtained in [8] and shares the same features as the Bian-Huang-Shen potential $V_{B H S}$ which depends only on odd powers of $r$ [22].
\end{abstract}

*mchabab@ucam.ac.ma 


\section{Introduction}

The axion $a$ and the dilaton $\phi$ are two particles predicted by superstring theories. They are among the probable candidates which could provide more informations about the Quantum Chromodynamics (QCD) vacuum [1, 2, 3]. The latter has a non trivial structure manifested by the presence of non-zero vacuum expectation values of certain operators. Theoretically, the axion was firstly introduced in QCD via the Peccei-Quinn mechanism where it appears as a pseudo-Goldstone boson resulting from the spontaneous breakdown of the $U(1)_{P Q}$ global symmetry in order to solve the strong $\mathrm{CP}$ problem [ [4]. This phenomenon is directly related to the presence of the topological term $\theta \frac{g^{2}}{32 \pi} G_{\mu \nu} \tilde{G}^{\mu \nu}$ violating both $\mathrm{P}$ and CP symmetries [4] and playing a crucial role in the Seiberg-Witten model [5]. Here $G_{\mu \nu}$ is the QCD field strength, $\tilde{G}_{\mu \nu}$ its dual and $\theta$ denotes the usual QCD vacuum angle. Furthermore, in cosmology, the axion is an hypothetical particle presented as a dark matter candidate and may constitute the missing mass of universe [6]. As to the dilaton, it is a scalar field appearing in several theories with different meanings, sometimes as a massless field and others as a massive one. In supersymmetric gauge theories, the dilaton $\phi$ appears as a companion of the axion: if the dilaton arises in the real part of the lowest component of a chiral superfield, the imaginary part corresponds to an axionic field. Within the Kaluza-Klein supergravity frame, if the field content of space-time is assumed to arise from embedding in $\left(4+d_{i}\right)$-dimensional manifold, the $d_{i}$ internal dimensions will have variable volume from the four-dimensional point of view and is interpreted as the dilaton. In string theory, the axion and the dilaton are in the masseless spectrum of 10D type IIB model and its lower dimensional compactifications. The field $\phi$ emerges from the NS-NS sector and $a$ from the RR sector and are at the basis of the discovery of F-theory and type IIB self duality [7]. Thus, there is no unique definition of the dilaton as it has various interacting ways; for instance, it couples naturally to super-Yang Mills gauge fields in curved space and plays a central role in string theory since it defines the string coupling constant $g_{s}$ as $e^{\phi}$. The dilaton has also been coupled to the Ricci curvature term as in the Brans-Dicke model of induced gravity as well as to the QCD field strength to generate the confining phase. In this case, it was shown in [8] that the coupling of a massive dilaton to gluon can generate a potential, of the quark-quark interactions, which exhibits a short range Coulomb potential plus a linear confining term at large distances. In this paper, we shall determine the QCD nonperturbative effects, involved in the axion potential and the interquark potential by the inclusion of dilatonic degrees of freedom. Specifically, we shall extend the Vecchia, Veneziano and Witten (VVW) 9, 10 model, of standard QCD in the chiral limit and for large colour number $N_{c}$, by introducing a string inspired effective dilaton-gluon coupling from which we 
shall derive both the axion and dilaton potentials. On the other hand, following [8], we shall re-analyze the Coulomb problem under the requirement that analytical solutions exist, other than Dick one. By making a simple assumption, a new solution for the dilaton-gluon coupling function will be determined, from which we shall generate an interquark potential which goes beyond Dick one and looks like the Bian-Huang-Shen potential [22]. The latter includes corrections from nonperturbative $\mathrm{QCD}$, encoded in the quarks and gluons vacuum condensates, and depends only on odd powers of interquark distance $\mathrm{r}$.

This paper is organized as follows: In Section 2, first we review the axion potential, using the gluodynamics effective model of Halperin and Zhitnitsky [11]. Then, we derive new solutions for the potential. In section 3, we study an extension of the VVW potential by considering the dilatonic effects. Section 4 is devoted to derive a new interquark potential with higher orders in $r$ using a massive dilaton field which couples to the YM kinetic term like $\frac{1}{F(\phi)} G_{\mu \nu} G^{\mu \nu}$. In section 5, we draw a general conclusion.

\section{Comments on the axion potential}

We start by recalling that the vacuum energy $E(\theta)$ can be expressed through the $\theta$-angle appearing in the $\mathrm{CP}$ violating term of QCD lagrangian as follows [12]:

$$
E(\theta)=E(0)-\frac{1}{2} \chi \theta^{2}
$$

where $\chi$ is the topological susceptibility given in terms of the light quark mass $m_{q}$ via the following formula [13] :

$$
\chi=i \int d x<0\left|T\left\{\frac{\alpha_{s}}{8 \pi} G \tilde{G}(x), \frac{\alpha_{s}}{8 \pi} G \tilde{G}(0)\right\}\right| 0>=\frac{m_{q}}{N_{f}}<0|\bar{\psi} \psi| 0>+O\left(m_{q}^{2}\right),
$$

with $N_{f}$ is the number of the flavors and $<0|\bar{\psi} \psi| 0>$ is the quark condensate.

Following [11], the one-to-one correspondence $\theta \rightarrow \frac{a}{f_{a}}$ allows us to derive the axion potential $\mathrm{V}(\mathrm{a})$ from Eq.(2.1),

$$
V(a)=V(0)-\frac{1}{2} \chi\left(\frac{a}{f_{a}}\right)^{2}
$$

where $f_{a}$ denotes the axion coupling constant. The equation (2.1) appears insufficient to describe interesting properties as it is not a periodic function of $\theta . \theta$ periodicity is an essential property to investigate vacuum features, especially for the derivation of the metastable states and the domain walls [14. 
A way to exhibit this periodicity in $\theta$ is to consider the Vecchia-Venezino-Witten (VVW) model of standard QCD. Its lagrangian in the chiral limit and for large colour number $N_{c}$ [9, 10 is given by,

$$
L=\frac{F_{\pi}}{2}\left(\operatorname{Tr}\left(\partial_{\mu} U \partial^{\mu} U^{+}\right)+\operatorname{Tr}\left(M U+M^{+} U^{+}\right)\right)+\frac{c}{N_{c}}(-i \log (\operatorname{det} U)-\theta)^{2}
$$

where $M$ is the mass matrix. By minimizing the potential of this model and taking the diagonal parameterization of the linear $\sigma$ model $\mathrm{U}$ matrix,

$$
U=\left(\begin{array}{ccc}
e^{-i \phi_{1}} & 0 & 0 \\
0 & e^{-i \phi_{2}} & 0 \\
0 & 0 & e^{-i \phi_{3}}
\end{array}\right)
$$

one find out that: (i) the obtained minima is invariant by shifts of $\theta$ as $\theta+2 \pi$; (ii) Physical observables are $\theta$ periodic [9, 10, 15].

An other way to derive the axion potential relies on the use of the Halperin-Zhitnitsky (HZ) model. In this model, one proceeds in two steps: the first step consists in building the gluodynamics potential while in the second step one uses the results of Veneziano-Yankielowicz (VY) supersymmetric model [16] as well as VVW model.

The gluodynamics potential is expressed as a Legendre transformation in terms of the sources $J$ and $\bar{J}$ and the so-called "glueball" fields $h$ and $\bar{h}$ via the following formula [11, 18]:

$$
-V \hat{U}(h, \bar{h})=W(h, \bar{h})+J \int d x h+\bar{J} \int d x \bar{h}
$$

where $V=\int d^{4} x$ is four dimensional volume, $\hat{U}(h, \bar{h})$ defines the glueballs potential and $W(h, \bar{h})$ is inherited from the action of the gluodynamics lagrangian consisting only of YM term $G_{\mu \nu} G^{\mu \nu}$ and the topological term $\theta \frac{g^{2}}{32 \pi} G_{\mu \nu} \tilde{G}^{\mu \nu}$. It obeys the following differential equations:

$$
\begin{aligned}
& \left.\frac{\partial^{n+1} W}{\partial J^{n+1}}\right|_{J=\bar{J}=0}=(-4)^{n} \int d x<H> \\
& \left.\frac{\partial^{n+1} W}{\partial \bar{J}^{n+1}}\right|_{J=\bar{J}=0}=(-4)^{n} \int d x<\bar{H}> \\
& \left.\frac{\partial^{l+k} W}{\partial J^{l} \partial \bar{J}^{k}}\right|_{J=\bar{J}=0}=0,
\end{aligned}
$$

which are simply a generalization of the low energy theorems written as two points functions $(n=1)$ :

$$
\begin{aligned}
& \lim _{q \rightarrow 0} i \int d x e^{i q x}<0|T\{H(x) H(0)\}| 0>=-4<H> \\
& \lim _{q \rightarrow 0} i \int d x e^{i q x}<0|T\{\bar{H}(x) \bar{H}(0)\}| 0>=-4<\bar{H}> \\
& \lim _{q \rightarrow 0} i \int d x e^{i q x}<0|T\{\bar{H}(x) H(0)\}| 0>=0,
\end{aligned}
$$


with $H=\left(G^{2}+\frac{i}{\xi} G \tilde{G}\right)$ and $\bar{H}=\left(G^{2}-\frac{i}{\xi} G \tilde{G}\right)$ and where $\xi$ is a parameter related to the topological susceptibility [11. Moreover, $W(h, \bar{h})$ can be expressed in terms of the glueball fields $h$ and $\bar{h}$ via the following relations:

$$
\begin{aligned}
& \frac{\partial W}{\partial J}=\int d x h \\
& \frac{\partial W}{\partial \bar{J}}=\int d x \bar{h}
\end{aligned}
$$

In order to give an explicit formula of the gluodynamics effective potential $\hat{U}(h, \bar{h})$, it is necessary to derive the expression of $W(h, \bar{h})$. Following [11], the following form given by:

$$
W(h, \bar{h})=-\frac{1}{4} \int d x<H>e^{-4 J}-\frac{1}{4} \int d x<\bar{H}>e^{-4 \bar{J}},
$$

satisfies (2.7) and leads to the effective potential,

$$
\hat{U}(h, \bar{h})=\frac{1}{4} \frac{q}{p} h \log \left(\frac{h}{C}\right)^{\frac{p}{q}}+\frac{1}{4} \frac{q}{p} \bar{h} \log \left(\frac{\bar{h}}{\bar{C}}\right)^{\frac{p}{q}}+D(h-\bar{h}),
$$

where the matrix elements $\langle H\rangle,\langle\bar{H}\rangle$ and the $\theta$ vacuum angle are encoded in $C, \bar{C}, D$ and in the glueball fields $h$ and $\bar{h}$.

Now we start the second step to derive the axion potential: We consider an effective potential inspired from the VY supersymmetric model which combines the gluodynamics potential (2.11) with the linear $\sigma$ model matrix $\mathrm{U}$ defined in (2.5):

$$
\begin{aligned}
e^{-i V W(h, U)}= & \Sigma_{n} \Sigma_{k} \exp \left\{-\frac{i V}{4}\left(\frac{p}{q} h \log \left(\left(\frac{h}{2 e E}\right)^{\frac{p}{q}} \operatorname{det} U\right)-2 \operatorname{Tr}(M U)+\text { h.c. }\right)\right. \\
& \left.+i \pi V\left(k+\frac{q}{p} \frac{\theta+2 \pi n}{2 \pi}\right) \frac{(h-\bar{h})}{2 i}\right\}
\end{aligned}
$$

with $E=b<\frac{\alpha_{s}}{32 \pi} G^{2}>$, and where $b$ is the first coefficient of the $\beta$-function and $p$ and $q$ are integers with $q / p \sim N_{c}$. Then, once the minimization procedure is performed, we obtain the following potential:

$$
W_{Q C D}\left(\theta, U, U^{+}\right)=E \cos \left(-\frac{p}{q}(\theta-i \log (\operatorname{det} U))\right)+\frac{1}{2} \operatorname{Tr}\left(M U+M^{+} U^{+}\right) .
$$

where $\rho$ and $\eta$ are physical fields appearing in the glueball field as $h=E e^{\rho+i \eta}$.

Note that the expansion of the cosine to the next-to-leading order in $\theta$ produces a part of the potential similar to VVW one. The latter is derived in the chiral limit and for large value 
of colour number $N_{c}$. By considering the missing terms at order up to $\frac{1}{N_{c}^{2}}$, we obtain the generalized formula of the vacuum energy:

$$
E(\theta)=E+\chi\left(\frac{q}{p}\right)^{2} \cos \left(\frac{p}{q} \theta\right)
$$

Finally, by invoking again the one-to-one correspondence: $\theta \rightarrow \frac{a}{f_{a}}$ and $E(\theta) \rightarrow V\left(\frac{a}{f_{a}}\right)$, the axion potential is derived,

$$
V(a)=-b<0\left|\frac{\alpha_{s}}{32 \pi} G^{2}\right| 0>+m_{q} N_{c}<0|\bar{\psi} \psi| 0>\cos \left(\frac{a}{f_{a} N_{c}}\right) .
$$

This expression exhibits an explicit $\theta$ periodicity as required and has the same form as a class of inflationary potential describing the vacuum [24]. At this stage, a couple of remarks are in order:

(i) we can obtain the same result as in (2.13) by an appropriate choice of $h$ and $\bar{h}$ incorporated in the following potential:

$$
\hat{U}(h, \bar{h})=\frac{1}{4} h \log \frac{h}{C^{\prime}}+\frac{1}{4} \bar{h} \log \frac{\bar{h}}{C^{\prime}}-i D^{\prime}(h-\bar{h}),
$$

where $C^{\prime}$ and $D^{\prime}$ are real. Indeed by taking $h=E e^{i D^{\prime}}$, a straightforward calculation provides the expression (2.13), with $C^{\prime}=\frac{E}{e}$ and $4 D^{\prime}=\frac{p}{q}(\theta-i \log (\operatorname{det} U))$ and where the glueball fields $h$ and $\bar{h}$ are parameterized via the $\theta$-angle and the $U$-matrix.

(ii) we also note that the system of differential equations (2.7) admits other solutions which are different from the HZ one (2.10). Consequently, other forms for $\hat{U}(h, \bar{h})$ can be obtained. In this paper, we propose the following alternative ones:

a) The first solution corresponds to:

$$
W(h, \bar{h})=-\frac{1}{4(n+1) !}(1-4 J)^{n+1} \int d x<H>-\frac{1}{4(n+1) !}(1-4 \bar{J})^{n+1} \int d x<\bar{H}>
$$

By putting back (2.17) in (2.6) and taking into account the constraints (2.7) and (2.9), the following equation emerges:

$$
\hat{U}-h \frac{\partial \hat{U}}{\partial h}-\bar{h} \frac{\partial \hat{U}}{\partial \bar{h}}=\frac{1}{8} \frac{h}{<H>}+\frac{1}{8} \frac{\bar{h}}{<\bar{H}>}
$$

for which the solution $\hat{U}(h, \bar{h})$ is given by:

$$
\hat{U}(h, \bar{h})=-\frac{1}{8} \frac{h^{2}}{<H>}+C_{1} h-\frac{1}{8} \frac{\bar{h}^{2}}{<\bar{H}>}+\bar{C}_{1} \bar{h} .
$$


b)The second solution reads as:

$$
W(h, \bar{h})=-\frac{1}{4(n+1) !} \frac{1}{(1+4 J)} \int d x<H>-\frac{1}{4(n+1) !} \frac{1}{(1+4 \bar{J})} \int d x<\bar{H}>,
$$

Similarly to (a), the constraints (2.7) and (2.9) lead to the differential equation:

$$
\hat{U}-h \frac{\partial \hat{U}}{\partial h}-\bar{h} \frac{\partial \hat{U}}{\partial \bar{h}}=\frac{1}{4} h^{1 / 2}<H>^{1 / 2}+\frac{1}{4} \bar{h}^{1 / 2}<\bar{H}>^{1 / 2},
$$

which admits the solution:

$$
\hat{U}(h, \bar{h})=\frac{1}{2} h^{1 / 2}<H>^{1 / 2}+C_{2} h+\frac{1}{2} \bar{h}^{1 / 2}<\bar{H}>^{1 / 2}+\bar{C}_{2} \bar{h},
$$

where $C_{1}$ and $C_{2}$ are complex constants.

Thus, like for HZ potential (2.11), we remark that the effective potentials (2.19) and (2.22) provide new ways to parameterize the glueballs interactions. The fact that there is no unique form of the glueballs potential is due to the complexity of the QCD vacuum structure which is encoded in the low energy theorems (2.8).

\section{The axion/dilaton Potentials}

As we have mentioned in the introduction of this paper, the dilaton and the axion are scalar fields predicted by string theory. In the language of the $4 \mathrm{D}$ gauge theory, the axion usually couples to the topological term while the dilaton couples to the field strength term. In this section, we shall exploit these features to build an effective potential in which we combine both of them. In this regard, we shall extend the lagrangian of VVW model [9, 10] by including the dilaton degrees of freedom,

$$
L_{V V W}=L_{0}\left(U, U^{+}\right)+L\left(A_{\mu}, \phi, \theta\right)+\frac{1}{8} i \epsilon_{\mu \nu \rho \sigma} G^{\mu \nu \rho \sigma} \operatorname{Tr}\left[\log U+\log U^{+}\right]+\frac{f_{\pi}}{2 \sqrt{2}} \operatorname{Tr}\left[M U+M U^{+}\right],
$$

This lagrangian is established at large $N_{c}$ limit in which QCD exhibits both confinement and chiral symmetry breaking. $L_{0}\left(U, U^{+}\right)$is a sigma model lagrangian which describes the low energy dynamics of the pseudoscalar mesons parameterized by the $U$ complex matrix:

$$
L_{0}\left(U, U^{+}\right)=\frac{1}{2} \operatorname{Tr}\left(\partial_{\mu} U \partial^{\mu} U^{+}\right) .
$$

The $\theta$-term in the QCD fundamental lagrangian will be substituted by $-\frac{\theta}{4} \epsilon_{\mu \nu \rho \sigma} G^{\mu \nu \rho \sigma}$, where $G^{\mu \nu \rho \sigma}$ is the field strength for a three-index field $A_{\mu \nu \rho}$ given by [9, 21]:

$$
G_{\mu \nu \rho \sigma}=\partial_{\mu} A_{\nu \rho \sigma}-\partial_{\sigma} A_{\mu \nu \rho}+\partial_{\rho} A_{\sigma \mu \nu}-\partial_{\nu} A_{\rho \sigma \mu}
$$


and where $A_{\mu \nu \rho}$ is related to the gauge field $A_{\mu}$ by the following expression:

$A_{\nu \rho \sigma}=\frac{g^{2}}{96 \pi^{2}}\left[-A_{\nu}^{a} \overleftarrow{\partial}_{\rho} A_{\sigma}^{a}+A_{\nu}^{a} \partial_{\rho} A_{\sigma}^{a}+A_{\rho}^{a} \overleftarrow{\partial}_{\nu} A_{\sigma}^{a}-A_{\rho}^{a} \partial_{\nu} A_{\sigma}^{a}+A_{\nu}^{a} \overleftarrow{\partial}_{\sigma} A_{\rho}^{a}-A_{\nu}^{a} \partial_{\sigma} A_{\rho}^{a}+2 f_{a b c} A_{\nu}^{a} A_{\rho}^{b} A_{\sigma}^{c}\right]$

$f_{a b c}$ are the $S U\left(N_{c}\right)$ structure constants.

Since the massive dilaton couples to the YM term $G_{\mu \nu} G^{\mu \nu}$ as follows:

$$
\mathcal{L}=-\frac{1}{4 F(\phi)} G_{\mu \nu} G^{\mu \nu}-\frac{1}{2} \partial_{\mu} \phi \partial^{\mu} \phi-\frac{1}{2} m^{2} \phi^{2}+J_{\mu}^{a} A_{a}^{\mu}
$$

we suppose that the kinetic term $-d G_{\mu \nu \rho \sigma} G^{\mu \nu \rho \sigma}$ of the field $A_{\nu \rho \sigma}$ in VVW model couples to the dilaton field via a given $\phi$ function $\frac{1}{F(\phi)}$. The form of $F(\phi)$ will be discussed in the next section. Then we write the second term in Eq.(3.1), which contains the gauge field $A_{\mu}$, the dilaton $\phi$ and the $\theta$-vacuum angle as :

$$
L\left(A_{\mu}, \phi, \theta\right)=\frac{-d}{F(\phi)} G_{\mu \nu \rho \sigma} G^{\mu \nu \rho \sigma}-\frac{\theta}{4} \epsilon_{\mu \nu \rho \sigma} G^{\mu \nu \rho \sigma}-\frac{1}{2} \partial^{\mu} \phi \partial_{\mu} \phi-\frac{1}{2} m^{2} \phi^{2},
$$

where $d$ is a positive constant and $m$ is the dilaton mass.

By considering the field $q(x)=\frac{1}{4} \epsilon_{\mu \nu \rho \sigma} G^{\mu \nu \rho \sigma}$, the effective lagrangian at large- $N_{c}$ reads as:

$$
\begin{aligned}
L_{V V W}\left(U, U^{+}, q, \phi\right)= & L_{0}\left(U, U^{+}\right)+\frac{1}{F(\phi)} N_{c} q^{2}(x)-\theta q(x)+\frac{1}{2} i q(x) \operatorname{Tr}\left[\log U+\log U^{+}(3.7)\right. \\
& +\frac{f_{\pi}}{2 \sqrt{2}} \operatorname{Tr}\left[M U+M U^{+}\right]-\frac{1}{2} \partial_{\mu} \phi \partial^{\mu} \phi-\frac{1}{2} m^{2} \phi^{2}
\end{aligned}
$$

Moreover, if The equation of motion of $\mathrm{q}(\mathrm{x})$ is used the expression (3.7) reduces to:

$$
\begin{aligned}
L\left(U, U^{+}, \phi\right)= & L_{0}\left(U, U^{+}\right)+\frac{c F(\phi)}{4 N_{c}}\left\{\theta-\frac{1}{2} \operatorname{Tr}\left[\log U+\log U^{+}\right]\right\}^{2} \\
& +\frac{f_{\pi}}{2 \sqrt{2}} \operatorname{Tr}\left[M U+M U^{+}\right]-\frac{1}{2} \partial_{\mu} \phi \partial^{\mu} \phi-\frac{1}{2} m^{2} \phi^{2}
\end{aligned}
$$

with $c=\frac{4 !}{d}$. For pure YM theory at large- $N_{c}$ coupled to the dilaton field, the effective potential $W$ containing $\theta$ and $\phi$ derived from the potential part of the lagrangian (3.8) is given by:

$$
W(\theta, \phi)=W(\theta=0, \phi)-\frac{c F(\phi)}{4 N_{c}} \theta^{2}-\frac{1}{2} m^{2} \phi^{2} .
$$

As usual, we adopt the guess that the missing orders in the $\frac{1}{N_{c}}$ expansion provide a cosine form for the potential $W(\theta, \phi)$, namely,

$$
W(\theta, \phi)=\frac{c F(\phi)}{4} N_{c} \cos \left(\frac{\theta}{N_{c}}\right)-\frac{1}{2} m^{2} \phi^{2} .
$$


Therefore, the axion potential derived via: $\theta \rightarrow \frac{a}{f_{a}}$ correspondence, can be written in terms of the dilatonic vacuum expectation value as:

$$
W(\theta, \phi)=\frac{c<F(\phi)>}{4} N_{c} \cos \left(\frac{\theta}{N_{c}}\right)-\frac{1}{2} m^{2}<\phi^{2}>.
$$

Such formula is more general: It describes the axion potential for any kind of dilaton-gluon coupling $F(\phi)$. If we consider the particular case $F(\phi)=\frac{\phi}{f}$, the dilaton mass and $<\phi^{2}>$ can be substituted in (3.11) by using the following relations [17]:

$$
\begin{aligned}
& m^{2} f_{\phi}^{2}=-\frac{2 \beta(g)}{g}<0\left|G_{\mu \nu} G^{\mu \nu}\right| 0>, \\
& 4<\phi^{2}>=-\frac{g}{2 \beta(g)} \frac{f_{\phi}^{3}}{f}-f_{\phi}^{2},
\end{aligned}
$$

with $-\frac{2 \beta(g)}{g}=\left(\frac{11}{3} N_{c}-\frac{2}{3} N_{f}\right) \frac{\alpha_{s}}{2 \pi}=b \frac{\alpha_{s}}{2 \pi}, f_{\phi}$ is the dilaton decay constant and $f$ represents a mass scale. The axion potential (3.11) then becomes

$$
W(a,<\phi>)=-\frac{1}{8} \frac{2 \beta(g)}{g}\left(1+\frac{g}{2 \beta(g)} \frac{f_{\phi}}{f}\right)<0\left|G_{\mu \nu} G^{\mu \nu}\right| 0>+\frac{c<F(\phi)>}{4} N_{c} \cos \left(\frac{a}{f_{a} N_{c}}\right),
$$

taking the same form as the $\mathrm{HZ}$ one, with the gluon vacuum condensate term expressed as an expansion at leading order in $\frac{f_{\phi}}{f}\left(\frac{f_{\phi}}{f} \ll 1\right)$. As a byproduct, For a vanishing vacuum angle, the formula (3.11) reduces to the expression of the dilaton potential,

$$
W(\phi)=\frac{c<F(\phi)>}{4} N_{c}-\frac{1}{2} m^{2} \phi^{2}
$$

The effective potentials given by Eqs.(3.9) and (3.12) generalize the VVW one which corresponds to the particular case when $F(\phi)=1$, the coupling of the gauge field to a massless dilaton. Consequently, the lagrangian $L\left(U, U^{+}, \phi\right)$ in (3.8) allows us to reconsider the $\mathrm{HZ}$ model by inclusion of the dilaton contribution.

\section{New Interquark Potential}

In QCD framework, quarks and gluons (in general all colored objects) appearing in the fundamental QCD lagrangian cannot exist as separate objects and then are absent from the physical spectrum: such phenomenon is explained by the confinement. Confinement in gauge theories provides one of the most challenging problems in theoretical physics. Various quark confinement models rely on flux tube picture. The latter emerges through the condensation of 
magnetic monopoles and explain the linear rising potential between color sources. However, a deep understanding of confinement mechanism in still lacking. Recently it has been shown in [8] that a string inspired coupling of a dilaton $\phi$ to the $4 \mathrm{~d} S U\left(N_{c}\right)$ gauge fields yields a phenomenologically interesting interquark potential $V_{D}(r)$ with a linear confining term. Extension of gauge field theories by inclusion of dilatonic degrees of freedom has gained considerable interest. Particularly, Dilatonic Maxwell and Yang Mills theories which, under some assumptions, possess stable, finite energy solutions [19]. Indeed, the derivation performed in 8 is remarkable since it provides a challenge to monopole condensation as a new quark confinement scenario. Therefore, our objective in this section is to dedicate more efforts to the investigation of this confinement generating mechanism with the aim to derive a new interquark potential, extending Dick one.

To this end, we propose an effective field theory described by the lagrangian,

$$
L=-\frac{1}{4 F(\phi)} G_{\mu \nu} G^{\mu \nu}-\frac{1}{2} \partial_{\mu} \phi \partial^{\mu} \phi-\frac{1}{2} m^{2} \phi^{2}+J_{\mu}^{a} A_{a}^{\mu},
$$

where the coupling $-\frac{1}{4 F(\phi)}$ is function of the dilaton field and $\mathrm{m}$ denotes the dilatonic mass. Such form appears in several theoretical frameworks, for instance: $\frac{1}{F(\phi)}=e^{\frac{\phi}{f_{\phi}}}$ as in string theory, $\frac{1}{F(\phi)}=\frac{\phi}{f_{\phi}}$ similar to the Cornwall-Soni model in which $\frac{1}{F(\phi)}$ parameterizes the coupling of gluon to the glueball field [18]; while in Dick model, $F(\phi)$ is given by: $\frac{1}{F(\phi)}=\frac{\phi^{2}}{f^{2}+\kappa \phi^{2}}$.

The analysis of the Coulomb problem of the dilatonic theory in (4.1) is performed by considering a point like static source described by the current density $J_{a}^{\nu}=g \delta(r) C_{a} \eta^{\mu}$ where $C_{a}$ is the expectation value of the $S U\left(N_{c}\right)$ generators for a normalized spinor in the color space , satisfying the algebra identity $\Sigma_{a} C_{a}^{2}=\frac{N_{c}^{2}-1}{2 N_{c}}$.

The equations of motion corresponding to $\phi$ and $A_{\mu}$, inherited from the lagrangian (4.1) and emerging from this source, read as,

$$
\begin{aligned}
& \partial^{2} \phi-m^{2} \phi=\frac{1}{4} \frac{d(1 / F(\phi))}{d \phi} G_{\mu \nu} G^{\mu \nu} \\
& \partial_{\mu}\left(\frac{1}{F(\phi)} G_{a}^{\mu \nu}\right)+g \frac{1}{F(\phi)} A_{\mu}^{b} f_{a b}^{c} G_{c}^{\mu \nu}=-J_{a}^{\nu},
\end{aligned}
$$

which yields

$$
\frac{d^{2}}{d r^{2}}(r \phi)-m^{2}(r \phi)=\frac{\mu^{2}}{2 r^{3}} \frac{d(F(\phi))}{d \phi}
$$

where the abbreviation $\mu=\frac{g}{4 \pi} \sqrt{\frac{N_{c}^{2}-1}{2 N_{c}}}$ is used. The equation (4.4) may be solved for a given 
dilaton-gluon coupling $F(\phi)$ for which the interquark potential $V(r)$ is expressed by the important formula [20]:

$$
V(r)=-\frac{g}{4 \pi} C \int \frac{F(\phi(r))}{r^{2}} d r
$$

Such form of potential is very attractive since it extends the usual Coulomb formula $V_{c}(r) \sim \frac{1}{r}$, recovered from (4.5) by taking $F(\phi)=1$. Moreover, for $F(\phi)=r^{n}$ with $n \geq 2$, the formula (4.5) yields a confining potential. On the other hand Eq.(4.5) may be also used to relate nonperturbative effects such as QCD vacuum condensates to dilaton parameters $(m, f)$ via comparison with known interquark potentials exhibiting QCD power corrections, such as BHS potential [22].

For Dick model $V_{D}(r)$ which corresponds to the coupling $F(\phi)=f^{2}+\frac{\kappa}{\phi^{2}}$, the confining potential is given by

$$
V_{D}(r)=\frac{N_{c}^{2}-1}{2 N_{c}}\left\{\frac{\kappa g^{2}}{4 \pi} \frac{1}{r}-g f \sqrt{\frac{N_{c}}{2\left(N_{c}-1\right)}} \log \left(e^{2 m r}-1+m y_{0}\right)\right\},
$$

Thus $V_{D}(r)$ exhibits two behaviors which characterize the quark interactions, namely: Besides Coulombian term which dominates at short distances, the potential develops, at large distances, a linear rising part, $V(r)=\sigma r$, describing the confining phase, where the string tension $\sigma$ is proportional to dilaton parameters $(\mathrm{m}, \mathrm{f})$ as $\sigma \sim 2 g \mathrm{fmr}$. Recently, Dick potential was successfully checked in 23] where it has been shown that, if the dilaton is assigned a mass about $57 \mathrm{MeV}$, the spectrum of the charmonium and bottomnium are reproduced well. Furthermore, the spin averaged energy levels of $B_{c}$ and $B_{c}^{*}$ systems have been estimated with values in good agreement with other theoretical framework predictions.

However, If we invoke the picture of the old string theory, confinement is connected to the formation of a string between two quarks (or quark-antiquark) separated by a distance $r$. If $r$ increases the string tension increases too. Then, for large distances the string may be broken and colored fields may appear as separate objects. In this regards, it is convenient to parameterize the confinement in another way which preserves color singlet feature of the system. To this end, we shall derive a more general confining potential than Dick one, where high powers in $r$ may appear. We rewrite the equation of motion (4.4) as follows:

$$
y^{\prime \prime}-m^{2} y=\frac{\mu^{2}}{2 r^{3}} \frac{d(F(\phi))}{d \phi},
$$

which, for Dick choice, reduces to:

$$
y^{\prime \prime}-m^{2} y=-\frac{\mu^{2}}{y^{3}}
$$


with $y=r \phi$ and $y^{\prime \prime}=\frac{d^{2} y}{d r^{2}}$.

Note that Eq.(4.7) can be interpreted as corresponding to a mechanical system with the action,

$$
S=\int d r L_{D}=\frac{1}{2} \int d r\left[\left(y^{\prime}\right)^{2}+m^{2} y^{2}+\frac{\mu^{2}}{r^{2}} F(y / r)\right]
$$

Consequently, the effective coupling $F(\phi)$ in (4.1) appears as a part of interacting potential of a 1d quantum field theory. In this case, looking for solutions of (4.8) is similar to solving the following equation of motion [20],

$$
y^{\prime \prime} \frac{\partial L_{D}}{\partial y^{\prime}}+y^{\prime} \frac{\partial L_{D}}{\partial y}+\partial_{r}{ }^{e x p} L_{D}=0
$$

Now in order to derive a potential with higher order in $r$, instead of the equation (4.7), let's consider,

$$
y^{\prime \prime}-m^{2} y=\mu^{2}\left(b_{0}+b_{1} r+b_{2} r^{2}\right)
$$

where the parameters $b_{0}, b_{1}$ and $b_{2}$ are arbitrary and are connected to the nonperturbative effects parameterized by the quark and gluon vacuum condensates, as it will be shown later. The explicit solution $\phi(r)=y / r$ of $(4.11)$ is

$$
\phi=\frac{1}{r}\left\{A e^{-m r}-\frac{\mu^{2}}{m^{2}}\left\{\left(b_{0}+\frac{2 b_{2}}{m^{2}}\right)+b_{1} r+b_{2} r^{2}\right\}\right\}
$$

where A is an integration constant. Substitution of Eq.(4.12) in (4.7) leads to the following expression of $F(\phi(r))$ as a function of $r$ :

$$
\begin{aligned}
F(\phi(r))= & K-\frac{2 \mu^{2}}{m^{2}}\left\{-\left(b_{0}+\frac{2 b_{2}}{m^{2}}\right) \frac{b_{0}}{2} r^{2}-\left(b_{0}+\frac{2 b_{2}}{m^{2}}\right) \frac{b_{1}}{3} r^{3}-\frac{b_{2}^{2}}{2 m^{2}} r^{4}+\frac{b_{1} b_{2}}{5} r^{5}+\frac{b_{2}^{2}}{6} r^{6}\right\} \\
& -\left\{\left(3 b_{0}+\frac{8 b_{1}}{m}+\frac{30 b_{2}}{m^{2}}\right) \frac{1}{m^{2}}+\left(3 b_{0}+\frac{8 b_{1}}{m}+\frac{30 b_{2}}{m^{2}}\right) \frac{r}{m}\right. \\
& \left.+\left(b_{0}+\frac{4 b_{1}}{m}+\frac{15 b_{2}}{m^{2}}\right) r^{2}+\left(b_{1}+\frac{5 b_{2}}{m}\right) r^{3}+b_{2} r^{4}\right\} A e^{-m r}
\end{aligned}
$$

Finally, by invoking the formula (4.5), we can easily obtain the interquark potential $V(r)$ :

$$
\begin{aligned}
V(r)= & \frac{\alpha K}{r}-\frac{2 \alpha \mu^{2}}{m^{2}}\left\{-\left(b_{0}+\frac{2 b_{2}}{m^{2}}\right) \frac{b_{0}}{2} r-\left(b_{0}+\frac{2 b_{2}}{m^{2}}\right) \frac{b_{1}}{6} r^{2}-\frac{b_{2}^{2}}{m^{2}} \frac{r^{3}}{6}+\frac{b_{1} b_{2}}{20} r^{4}+\frac{b_{2}^{2}}{30} r^{5}\right\} \\
& -\alpha A e^{-m r}\left\{\left(3 b_{0}+\frac{8 b_{1}}{m}+\frac{30 b_{2}}{m^{2}}\right) \frac{1}{m^{2} r}+\left(b_{0}+\frac{5 b_{1}}{m}+\frac{22 b_{2}}{m^{2}}\right) \frac{1}{m}\right. \\
& \left.+\left(b_{1}+\frac{7 b_{2}}{m}\right) \frac{r}{m}+\frac{b_{2}}{m} r^{2}\right\} .
\end{aligned}
$$


where $\alpha=\frac{g}{4 \pi} C$. This form of potential containing higher power of $r$ is very interesting thanks to the presence of several terms which come with different signs and have not the same behaviours: Indeed, since some terms provide a confinement effect while the others have a deconfinement effect, the total interquark potential should not diverge for large $r$. This potential can be compared with the Bian-Huang-Shen potential $V_{B H S}$ calculated in terms of the QCD vacuum condensates in the QCD background fields [22]

$$
V_{B H S}(r)=\left(-\frac{4}{3 \pi} g^{2}+B_{f}\right) \frac{1}{r}+D r+F r^{3}+G r^{5}+\left(-\frac{B_{f}}{r}+C_{f} r+F_{f} r^{2}\right) e^{-m_{f} r}
$$

where the parameters $B_{f}, C_{f}, D, E_{f}, F$ and $G$ are expressed through the following condensates (up to dimension 6) $<0|\bar{\psi} \psi| 0>,<0\left|\frac{\alpha}{\pi} G^{2}\right| 0>,<0|\bar{\psi} \sigma G \psi| 0>,<0\left|\bar{\psi} \Gamma_{1} \psi \bar{\psi} \Gamma_{2} \psi\right| 0>$ and $f_{a b c}<0\left|G_{a}^{\mu \nu} G_{\nu \rho}^{b} G_{\mu c}^{\rho}\right| 0>$. Moreover, If we assume that terms with an exponential behaviour have a negligible contribution, in both potentials $V(r)$ and $V_{B H S}(r)$ (this corresponds to the behaviour of the potentials at large distances) with $b_{1}$ sets to zero in Eq.(4.14), we will see that only terms with odd powers in $r$ survive. This result is in perfect agreement with the BHS hypothesis suggesting that for higher dimension condensates, the interquark potential can be written as:

$$
V(r)=-\frac{\alpha}{r}+\Sigma_{n}(-1)^{n} C_{2 n+1} r^{2 n+1} .
$$

depending only on odd powers of $r$ with alternating signs and where the coefficients $C_{2 n+1}$ are function of the QCD condensates. At the same time, the confrontation of $V(r)$ with $V_{B H S}(r)$ (resp. $V_{D}(r)$ generates several relations between $\left(b_{0}, b_{1}, b_{2}\right)$ (resp. $(f, m)$ ) and the vacuum condensates which may provide an important piece of information in the search of the dilaton.

\section{Conclusion}

In this paper we have reviewed the derivation procedure of the axion potential as given by $\mathrm{HZ}$ and have proposed the alternative forms for $U(h, \bar{h})$ gluodynamics effective potentials. We have seen that for the HZ solution, we can obtain the axion potential as it is written in Eq.(2.13) directly by setting the glueball field $h$ in Eq.(2.16) as: $h=E e^{i D^{\prime}}$. As a byproduct, we have found that the physical field $\eta$ is expressed in terms of the $\theta$ vacuum and the pseudo-Goldstone fields given by the $U$ matrix. The axion potential in (2.15), which is parameterized by the vacuum condensates, has the same form as a class of inflationary potentials describing the vacuum potential, generally deduced by invoking the inflaton field [24]. Next, we have extended the VVW model by the implementation of the dilaton field. The new VVW-lagrangian allows us to derive the dilaton potential and to regenerate the axion 
one which take into account dilatonc degrees of freedom. On the other hand, as the QCD vacuum has a non trivial structure and the physical spectrum does not contain colored objects, many potential models have been suggested to describe the confining phases characterizing the quark-quark interactions. We have derived in (4.14) a new interquark potential containing terms with high power of $r$. The latter emerges from a string inspired model with an effective dilaton-gluon coupling. The good agreement between $\mathrm{V}(\mathrm{r})$ and $V_{B H S}(r)$, in the particular case of $b_{1}=0$, allows us to adopt the general expression (4.16) with only odd powers of $r$ and alternating signs as an adequate confining potential form for higher dimension vacuum condensates.

\section{Acknowledgments}

We are grateful to Prof. J. da Providencia for his collaboration in the early stage of this work. We also wish to thank Prof. T. Lhallabi for discussions.

This work is supported by the program PARS/ 27.372/98/CNR and the CNPRST/ICCTI convention 340/00 CNR.

\section{References}

[1] T. Hatsua, and T. Kunihiro, Phys. Rep., Vol. 247, (1994) 221.

[2] D. H. Lyth and A. Riotto, Phys. Rep. 314 (1999) 1.

[3] R. Dick, Phys. Lett. B397 (1997) 193.

[4] R. D. Peccei and H. R. Quinn, Phys. Rev. D16 (1977) 1791.

[5] N. Seiberg and E. Witten, Nucl. Phys. B426 (1994) 19.

[6] A. Sakharov, JETP Lett. 5 (1967) 24.

[7] C. Vafa, Nucl. Phys. B469 (1996) 403; J. H. Schwartz, Phys. Lett. B360 (1995) 13.

[8] R. Dick, Euro. Phys. J. C6 (1999) 701.

[9] P. D. Vecchia and G. Veneziano, Nucl. Phys. B171 (1980) 253.

[10] E. Witten, Annals Phys. 128 (1980) 363.

[11] I. Halperin and A. Zhitnitsky, Phys. Rev. D58 (1998); Phys. Lett. B440 (1998) 77. 
[12] R. J. Crewther, Phys. Lett. B70 (1977) 349.

[13] M. A. Shifman, A. I. Vainshtein and V. I. Zakharov, Nucl. Phys. B166 (1979) 493.

[14] G. Dvali, A. Melfo and G. Senjanovic, Phys. Rev. D54 (1996) 7857.

[15] A. Smilga, Phys. Rev. D29 (1984) 1424.

[16] G. Veneziano and S. Yankielowicz, Phys. Lett. B113 (1982) 231; Nucl. Phys. B218 (1983) 439.

[17] R. Dick and L.P. Fulcher, Euro. Phys. J. C9 (1999) 271.

[18] J. M. Cornwall and A. Soni, Phys. Rev. D29(1994) 1224.

[19] M. Cvetic and A.A. Tseytlin, Nucl. Phys. B171 (1980) 253.

[20] M. Chabab, R. Markazi and E. H. Saidi, Euro. Phys. J. C13 (2000) 543; M. Chabab, "Comments on the confinement from dilaton-gluon coupling in QCD", hep-th/0009115.

[21] G. Gabadadze and M. Shifman, Phys. Rev. D62 (2000)114003 .

[22] J. G. Bian, T. Huang and Q. X. Shen, Commun. Theor. Phys. 21 (1994) 333 and references therein.

[23] T. Barakat and M. Chabab, "Heavy meson masses via Dick interquark potential ", hepph/0101056.

[24] W. H. Kinney and K. T. Mahanthappa, Phys. Rev. D53 (1996) 5455; M. Brisudova and K.T. Mahanthappa, Phys. Rev. D62 (2000) 103516. 